\title{
FAST BEAM-ION INSTABILITY SIMULATIONS IN THE TESLA ELECTRON DAMPING RING AND THE FEL BEAM TRANSFER LINE
}

\author{
C. Montag, DESY, Hamburg, Germany* $\dagger$
}

\section{Abstract}

The Fast Beam-Ion Instability is considered potentially harmful in electron storage rings and linear colliders with short bunch spacing and high bunch charge, as it is the case in the proposed electron damping ring and the FEL beam transfer line of the future linear collider TESLA. This instability arises from interaction between a stored bunch and an ion cloud previously created by all heading bunches during a single pass. To study this effect and to determine the required vacuum conditions, a simulation code has been developed. The results of these simulation studies are presented in this paper.

\section{INTRODUCTION}

The Fast Beam-Ion Instability (FBII) [1] arises from the interaction of an electron bunch with the ion cloud generated by all heading bunches in the train during a single pass. When the bunch center is transversally displaced with respect to the center-of-mass of the ion cloud, both the ions and the bunch receive a transverse kick. If the coupling between bunches due to the ion cloud is strong enough to counteract the damping due to synchrotron radiation or transverse feedback systems, this might lead to large bunch oscillation amplitudes.

As long as the number of molecules in the beam path is large compared to the number of ions created by the beam, the number of ions created during a single bunch passage is proportional to the bunch charge. The initial transverse distribution of the ions equals the transverse electron distribution in the bunch. In the gap between bunches the ions drift freely, leading to a transverse blow-up of the ion cloud. Therefore, the larger the distance $L_{\text {sep }}$ between bunches, the weaker the bunch-to-bunch coupling. For this reason, the Fast Beam-Ion Instability is expected to occur in high-current, low-emittance machines with a large number of bunches, such as linear collider damping rings or the TESLA FEL beam transfer line.

To study the effect of the Fast Beam Ion Instability in the TESLA electron damping ring as well as the TESLA FEL beam transfer line, a simulation code has been developed. This paper describes the simulation method as well as results, leading to an estimate of the required vacuum pressure.

\section{SIMULATION METHOD}

Under the assumption of a transverse gaussian distribution of the electron bunch, the interaction between bunch

\footnotetext{
*montag@mail.desy.de

$\dagger$ Now at BNL, Upton, NY 11973
}

and ions can be described in analogy with the beam-beam interaction. The change in transverse velocity of the $i$ th ion due to interaction with the electron bunch is therefore given as

$$
\Delta v_{y, i}+i \Delta v_{x, i}=-2 N_{b} r_{e} c \frac{m_{e}}{M_{i}} f\left(x_{i}, y_{i}\right),
$$

where $f(x, y)$ is given by the well-known Bassetti-Erskine formula [2] as

$$
\begin{array}{r}
f(x, y)=-\sqrt{\frac{\pi}{2\left(\sigma_{x}^{2}-\sigma_{y}^{2}\right)}}\left[w\left(\frac{x+i y}{\sqrt{2\left(\sigma_{x}^{2}-\sigma_{y}^{2}\right)}}\right)\right. \\
-\exp \left(-\frac{x^{2}}{2 \sigma_{x}^{2}}-\frac{y^{2}}{2 \sigma_{y}^{2}}\right) \\
\left.w\left(\frac{x \frac{\sigma_{y}}{\sigma_{x}}+i y \frac{\sigma_{x}}{\sigma_{y}}}{\sqrt{2\left(\sigma_{x}^{2}-\sigma_{y}^{2}\right)}}\right)\right] .
\end{array}
$$

Here

$$
w(z)=\exp \left(-z^{2}\right)[1-\operatorname{erf}(-i z)]
$$

with

$$
\operatorname{erf}(x)=\frac{2}{\sqrt{\pi}} \int_{0}^{\pi} \exp \left(-t^{2}\right) \mathrm{d} t
$$

denotes the complex error function. $N_{b}, r_{e}, c, m_{e}$, and $M_{i}$ are the number of electrons per bunch, the classical electron radius, the velocity of light, the electron rest mass and the ion rest mass, while $x_{i}, y_{i}$ denote the transverse distances of the ion with respect to the bunch center.

For simulation purposes, the ion cloud is represented by macroparticles. This macroparticle representation has to be taken into account when calculating the kick on the electron bunch from the reaction force,

$$
\Delta y^{\prime}+i \Delta x^{\prime}=\frac{2 N_{b} r_{e}}{\gamma} \sum_{i} N_{i} \cdot f\left(x_{i}, y_{i}\right),
$$

where $\gamma$ and $N_{i}$ denote the relativistic factor of the electron bunch and the number of ions represented by the $i$ th macroparticle, respectively.

The number of ionization points is chosen to be equal to the number of optics elements of the accelerator lattice. At each of these ionization points,

$$
N_{\text {ions }}=N_{b} \cdot \sigma_{\text {ion }} \cdot n_{\text {mol }}
$$

ions are created per bunch passage, where $\sigma_{\text {ion }}$ denotes the ionization cross section. The area density of residual gas molecules, $n_{\mathrm{mol}}$, is computed from the vacuum pressure 


\begin{tabular}{|l|c|c|c|}
\hline & & damping ring & transfer line \\
\hline energy & $E / \mathrm{GeV}$ & 5.0 & 35.0 \\
damp. time & $\tau / \mathrm{msec}$ & 28 & - \\
hor. emitt. & $\epsilon_{x} / \mathrm{m}$ & $1 \cdot 10^{-9}$ & $1.5 \cdot 10^{-11}$ \\
vert. emitt. & $\epsilon_{y} / \mathrm{m}$ & $2 \cdot 10^{-12}$ & $1.5 \cdot 10^{-11}$ \\
$\# \mathrm{e}^{-} /$bun. & $N_{b}$ & $2 \cdot 10^{10}$ & $6.25 \cdot 10^{9}$ \\
bunch spac. & $L_{\mathrm{sep}} / \mathrm{m}$ & 6.0 & 30.0 \\
\hline
\end{tabular}

Table 1: Parameters of the TESLA damping ring and the FEL transfer line.

$p$ and the length $L_{j}$ of the particular optics element under consideration as

$$
n_{\mathrm{mol}}=L_{j} \cdot 6.023 \cdot 10^{26} \cdot \frac{p}{1.013 \mathrm{bar}} .
$$

Initially the first bunch is transversally displaced, while all consecutive bunches start on the closed orbit. The bunch train is tracked from one ionization point to the other along the accelerator using linear optics. At each ionization point each bunch creates $N_{\text {ions }}$ ions that are represented by $N_{\text {macro }}$ macroparticles. The initial transverse distribution of these macroparticles equals the transverse distribution of electrons in the bunch. The $N$ th bunch interacts with all $(N-1) \cdot N_{\text {ions }}$ ions created by the heading $N-1$ bunches. The resulting change in transverse velocity is computed according to Equation 1, while the kicks on the bunch are expressed according to Equation 5. After the passage of the $N$ th bunch the new ion coordinates and and velocities for all ions at the arrival time of the $(N+1)$ st bunch are calculated as

$$
\begin{array}{r}
v_{z, N+1}=v_{z, N}+\Delta v_{z, N}, \\
z_{N+1}=z_{N}+v_{z, N+1} \cdot \Delta t,
\end{array}
$$

where $z=x, y$, respectively, and $\Delta t=L_{\mathrm{sep}} / c$ is the bunch spacing.

\section{RESULTS}

For these simulations, an ionization cross section of 2 Mbarn for nitrogen gas with a molecular mass number $A_{N_{2}}=28$, corresponding to a molecular mass of $M_{N_{2}}=28 \cdot 938 \mathrm{MeV} / c^{2}$, has been assumed [3]. The ion cloud created by a single bunch at each ionization point is represented by 52 macroparticles. Some parameters of both beam lines are given in Table 1 .

\subsection{FBII in the TESLA electron damping ring}

The TESLA electron damping ring is basically composed of two arc sections with an average bending radius of $145 \mathrm{~m}$, and two $7.5 \mathrm{~km}$ long straight sections [4]. Each arc consists of $60 \mathrm{TME}$ cells, while each straight section is composed of 70 identical FODO cells. To limit the total number of macroparticles to be handled, only the first 20 bunches in the bunch train have been considered. The first bunch is transversally displaced by $\Delta x=0.1 \sigma_{x}$, $\Delta y=0.1 \sigma_{y}$.

For a vacuum pressure of $1 \cdot 10^{-8}$ mbar the vertical oscillation amplitude of the 5 th bunch equals the vertical rms beam size after one damping time (500 turns), while the corresponding value for consecutive bunches is even larger, see Figure 1.

When the vacuum pressure is reduced by an order of mag-

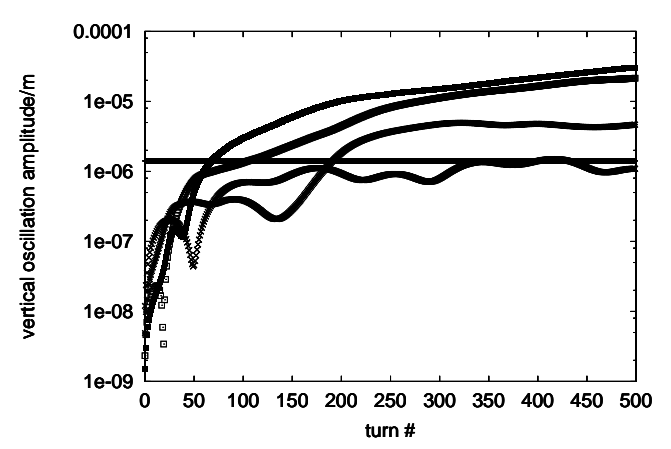

Figure 1: Oscillation amplitude of (from bottom to top) the 5 th, the 10 th , the 15 th and the 20 th bunch in the bunch train for a vacuum pressure of $1 \cdot 10^{-8}$ mbar. The horizontal straight line corresponds to the vertical beam size.

nitude to $p=1 \cdot 10^{-9}$ mbar, the oscillation amplitude of trailing bunches increases much more slowly. After 500 turns, the vertical oscillation amplitude of the 5th bunch corresponds to only a tenth of the vertical rms beam size, while it is even smaller for consecutive bunches, see Figure 2.

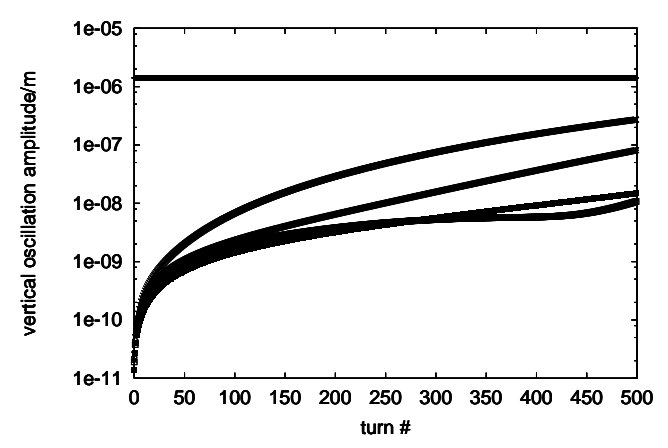

Figure 2: Oscillation amplitude of (from top to bottom) the 5 th, the 10th, the 20th, and the 15th bunch in the bunch train for a vacuum pressure of $1 \cdot 10^{-9} \mathrm{mbar}$. The horizontal straight line corresponds to the vertical beam size.

\subsection{FBII in the FEL transfer line}

The $12 \mathrm{~km}$ long TESLA FEL beam transfer line consists of 80 FODO cells composed of permanent magnets of identical strength [6]. Its design energy is $35 \mathrm{GeV}$, but 
the beam line provides sufficient flexibility to suit beam energies between 15 and $50 \mathrm{GeV}$. The simulations presented here were performed for the $35 \mathrm{GeV}$ case for which the phase advance per FODO cell is $42^{\circ}$. Since beam emittances as well as optics parameters are very similar in both planes, it is sufficient to investigate one single plane.

Initially, the first bunch is (vertically) displaced by $\Delta y=$ $0.1 \sigma_{y}$, while all trailing bunches start on the design trajectory. Figure 3 depicts the vertical oscillation amplitude of 100 bunches in a train at the end of the FEL beam transfer line for a vacuum pressure of $p=1 \cdot 10^{-6} \mathrm{mbar}$. All resulting oscillation amplitudes remain far below the vertical beam size and are therefore tolerable.

When the residual gas pressure is increased to $p=$

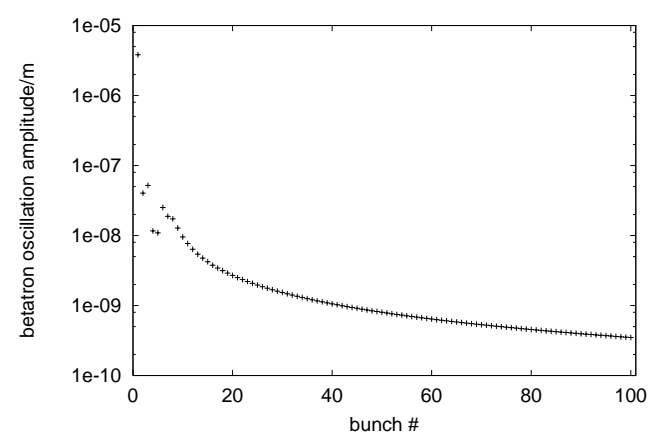

Figure 3: Vertical betatron oscillation amplitudes of 100 bunches at the end of the FEL transfer line for a vacuum pressure of $1 \cdot 10^{-6} \mathrm{mbar}$. The vertical beam size is indicated by the oscillation amplitude of the first bunch.

$1 \cdot 10^{-5}$ mbar, the oscillation amplitudes of several bunches in the head of the bunch train reach around $0.3 \sigma_{y}$, see Figure 4. For the rest of the bunch train the oscillation amplitudes are much smaller. To investigate the possibility of

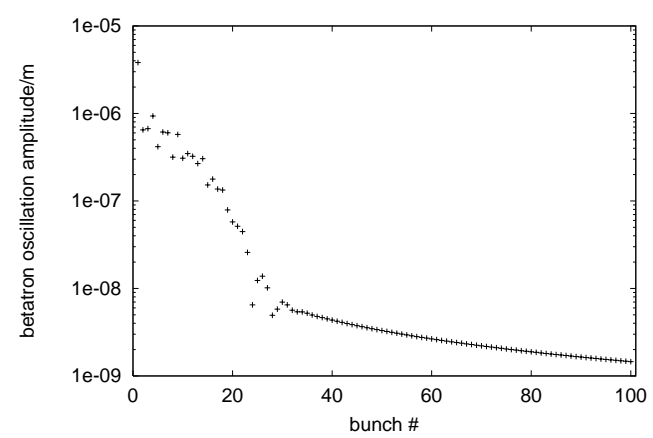

Figure 4: Vertical betatron oscillation amplitudes of 100 bunches at the end of the FEL transfer line for a vacuum pressure of $1 \cdot 10^{-5} \mathrm{mbar}$. The vertical beam size is indicated by the oscillation amplitude of the first bunch.

an ion-driven head-tail instability, a single bunch was cut into five slices. The first slice was initially displaced by
$\Delta x=0.1 \sigma_{x}, \Delta y=0.1 \sigma_{y}$. In this case an almost linear increase of the betatron oscillation amplitude of the following slices along the beam line is observed in the simulations. During the passage of a single bunch of only $25 \mu \mathrm{m}$ length, the ions practically do not change their position. Therefore only ions created by the first slice lead to amplitude growth. At the end of the transfer line the oscillation amplitude of the trailing slices corresponds to $0.05 \sigma$ for a vacuum pressure of $p=1 \cdot 10^{-5} \mathrm{mbar}$, and $0.005 \sigma$ for the $p=1 \cdot 10^{-6}$ mbar case.

Starting with a "banana" shaped bunch with the initial displacement of the $i$ th slice being

$$
\Delta z_{i}=a_{0} \cdot \sigma_{z} \cdot\left[1-\cos \left(\frac{2 \pi \cdot s_{i}}{8 \cdot l_{\text {bunch }}}\right)\right],
$$

where $l_{\text {bunc h }}=s_{5}-s_{1}$ and $s_{i}$ are the bunch length and the longitudinal position of the slice with respect to the head of the bunch, respectively, practically no change in the oscillation amplitude occurs along the transfer line for $a_{0}$ in the range $0.1 \ldots 0.5$.

\section{CONCLUSION}

The simulation studies presented in this paper indicate that a vacuum pressure of $p=1 \cdot 10^{-9}$ mbar in the TESLA electron damping ring and $p=1 \cdot 10^{-6}$ mbar in the TESLA FEL beam transfer line are sufficient to avoid the Fast Beam-Ion Instability. Both pressures can be achieved with standard methods.

\section{ACKNOWLEDGEMENTS}

I would like to thank R. Brinkmann and W. Decking for stimulating discussions.

\section{REFERENCES}

[1] T. O. Raubenheimer, F. Zimmermann, Phys. Rev. E 52 (1995) 5487

[2] M. Bassetti, G. Erskine, CERN-ISR-TH/80-06

[3] A.G. Mathewson, O.Groebner, Thermal Outgassing and Beam Induced Desorption, in: A. Chao, M. Tigner (eds.), Handbook of Accelerator Physics and Engineering, World Scientific, 1999

[4] K. Floettmann, J. Rossbach, DESY 93-023

[5] W. Decking, New Dog-bone Damping Ring Lattice, www.desy.de/wdecking/dog/dogbone.html

[6] G. Wüstefeld, Transferline (TFL), presentation at the TESLA TDR meeting February 2000 (unpublished) 